\title{
Personalsituation auf neonatologischen und pädiatrischen Intensivstationen - eine europaweite Umfrage
}

\section{Medical Staffing in Neonatal and Pediatric Intensive Care Units - an European Survey}

\author{
Autoren \\ Jörg Michel, Michael Hofbeck, Lisa Gerster, Felix Neunhoeffer
}

\section{Institut}

Department of Pediatric Cardiology, Pulmology and Pediatric Intensive Care Medicine, Universitätsklinikum Tübingen, Tübingen

\section{Schlüsselwörter}

Pflegenotstand, Geburtshilfe, Neonatologie, Intensivstation, Herzchirurgie, Ärztemangel

\section{Key words}

Nursing crisis, PICU, NICU, Neonatology, Lack of physicians, obstetrics

\section{Bibliografie}

DOI https://doi.org/10.1055/a-0991-0336

Klin Padiatr 2019; 231: 255-261

(c) Georg Thieme Verlag KG Stuttgart · New York ISSN 0300-8630

\section{Korrespondenzaddresse}

Dr. Jörg Michel

Department of Pediatric Cardiology

Pulmology and Pediatric Intensive Care Medicine

University Children's Hospital Tübingen

Hoppe-Seyler-Straße 1

72076 Tübingen

Tel.: +49/707/12983781, Fax: +49/707/1290

joerg.michel@med.uni-tuebingen.de

Den Anhang finden Sie unter https://doi.org/10.1055/a0991-0336

\section{ZUSAMMENFASSUNG}

Hintergrund In den vergangenen Jahren zeichnet sich ein zunehmender Fachkräfte- und Pflegemangel im Gesundheitswesen der Bundesrepublik Deutschland ab. Insbesondere im hochspezialisierten Bereich der neonatologischen und pädiatrischen Intensivmedizin kann dies zu Versorgungsengpässen führen.

Methode Wir führten eine Umfrage zur Erfassung der Personalsituation auf 583 neonatologischen und pädiatrischen Intensivstationen in Europa durch.
Ergebnisse Die Rücklaufquote betrug 48,5\%. Als wesentliches Ergebnis zeigte sich in Deutschland eine geringere Stellenbesetzung im pflegerischen Bereich im Vergleich zu den anderen teilnehmenden Ländern (90,4\% gegenüber 95,8\%, $\mathrm{p}<0,001)$. Zudem werden in Deutschland durchschnittlich mehr neonatologische Patienten pro Pflegekraft versorgt (3,0 gegenüber 2,3, $p<0,001$ ). Die Anwesenheit von Fach- und Oberärzten ist auf deutschen Intensivstationen werktags (12,0 h gegenüber $14,6 \mathrm{~h}, \mathrm{p}=0,04)$ und am Wochenende $(8,9 \mathrm{~h}$ gegenüber $13,2 \mathrm{~h}, \mathrm{p}=0,003$ ) geringer als in den anderen Ländern, wobei länderübergreifend der Wunsch nach einer längeren Facharztanwesenheit besteht.

Diskussion Unsere Ergebnisse zeigen eine im internationalen Vergleich schlechtere Besetzung von Pflegestellen auf neonatologischen und pädiatrischen Intensivstationen in Deutschland. Der dadurch drohende Qualitätsverlust in der Patientenversorgung wird dadurch verstärkt, dass im Vergleich in Deutschland vergleichsweise weniger Zeit durch fach- und oberärztliche Anwesenheit abgedeckt wird. Mögliche Folgen sind Zunahme von Komplikationen mit Gefährdung des Behandlungserfolgs, Versorgungsengpässen und psychischen sowie physischen Erkrankungen der Mitarbeiter führen.

Schlussfolgerung Im internationalen Vergleich zeigt sich ein Fachkräftemangel auf neonatologischen und pädiatrischen Intensivstationen in Deutschland. Dies unterstreicht die Notwendigkeit von energischen Maßnahmen zur Beseitigung des Pflegenotstands und Prävention eines drohenden Ärztemangel.

\section{ABSTRACT}

Background Recently, there is a growing scarcity of qualified personnel in German healthcare system. This can lead to problems especially in the highly specialized neonatal and pediatric intensive care.

Methods We conducted a survey to evaluate the staffing situation at 583 European neonatal and pediatric intensive care units (ICU).

Results Response rate was $48.5 \%$. As a main result our evaluation demonstrated a significant lower nurse-staffing in Germany compared to the other countries ( 90.4 vs. $95.8 \%, p<0.001)$. In addition, the average nurse-to-patient ratio was worse in German neonatal ICUs (3.0 vs. 2.3, $\mathrm{p}<0.001$ ). The presence of seni- 
or doctors is also lower in German neonatal and pediatric ICUs compared to the other countries (on weekdays: $12.0 \mathrm{vs.} 14.6 \mathrm{~h}$, $\mathrm{p}=0.04$; on weekends: 8.9 vs. $13.2 \mathrm{~h}, \mathrm{p}=0.003$ ).

Discussion Our survey demonstrates that the nurse-staffing at neonatal and ICUs in Germany is worse than in other European countries. The resulting potential loss of quality is worsened due to lower presence of senior doctors at the ICUs. Possi- ble consequences include increase of complications, impairment of treatment success, closure of intensive care beds, and mental and physical illness of the staff.

Conclusion Compared to other European countries, there is a lack of nurses at neonatal and pediatric ICUs in Germany. This study underlines the importance to take measures to counteract nursing crisis.

\section{Einleitung}

Die Perinatalmedizin und pädiatrische Intensivmedizin stellen aufgrund der Vielfalt aber auch der Besonderheiten der neonatologischen und pädiatrischen Krankheitsbilder sowie des breiten Behandlungsspektrums von Frühgeborenen mit wenigen hundert Gramm Geburtsgewicht bis zum fast erwachsenen Patienten hohe Anforderungen an die Struktur und Ausstattung der Intensivstationen. Dies betrifft v. a. auch die Qualifikation und Kompetenz des pflegerischen und ärztlichen Personals.

Es gibt Studien, die belegen, dass der Pflegeschlüssel und die pro Patienten verfügbaren Pflegestunden mit dem Behandlungserfolg korrelieren. Dies ist im Erwachsenenbereich sowohl für Normalstationen [1, 7, 10] als auch für Intensivstationen [4, 14, 20, 29] untersucht. Aber auch für die intensivmedizinische Betreuung kinderkardiochirurgischer Patienten gibt es Daten, dass ein höherer Anteil an Pflegepersonal und mehr Pflegestunden pro Patient die Rate an fehlgeschlagenen Extubationsversuchen nach herzchirurgischen Eingriffen reduzieren können [23]. In der Neonatologie wurde gezeigt, dass eine hohe Arbeitsbelastung der Pflegekräfte mit einer Vernachlässigung der Patientenpflege [26] und ein höheres Verhältnis von Pflegekräften je Patienten mit einem geringeren Risiko für ein schlechtes Outcome bei extrem Frühgeborenen assoziiert sind [2].

Um dem entgegen zu wirken, verabschiedete in Deutschland der Gemeinsame Bundesausschuss (G-BA) Richtlinien unter anderem für die Neonatologie und Kinderherzchirurgie, die die strukturellen, organisatorischen, personellen und fachlichen Anforderungen an die Versorgung der jeweiligen Patientengruppe vorgeben $[8,9]$. Eine G-BA Richtlinie dediziert für die pädiatrische Intensivmedizin gibt es zum aktuellen Zeitpunkt nicht. Neben den erwähnten G-BA Richtlinien gibt es zudem von verschiedenen Fachgesellschaften und Experten Leitlinien, die ebenfalls zur Qualitätssicherung auf den Intensivstationen beitragen sollen [15, 21, 24, 27].

In Bezug auf die bestehenden Richt- und Leitlinien konnten die Kollegen Warncke et al. im deutschsprachigen Raum bereits strukturelle und organisatorische Defizite auf pädiatrischen Intensivstationen feststellen [28]. Das Ziel dieser vorliegenden Untersuchung war es nun, insbesondere die personellen Begebenheiten sowohl auf neonatologischen als auch auf pädiatrischen Intensivstationen in mehreren europäischen Ländern zu erfassen und die Situation in deutschen Kliniken mit den Verhältnissen im europäischen Ausland zu vergleichen.

\section{Methoden}

Wir erstellten mithilfe einer webbasierten Software (Lime-Survey, https://www.limesurvey.org) eine Online-Umfrage, die in 3 verschiedene Sprachen übersetzt wurde (Deutsch, Englisch und Französisch). Diese wurde im März 2018 per E-Mail an insgesamt 583 Leitungen (ärztlich und/oder pflegerisch) von neonatologischen und pädiatrischen Intensivstationen in Deutschland (309), Österreich (32), der Schweiz (23), England (168), Frankreich (32) und Norwegen (19) versandt. Die Kontaktdaten wurden über die Webseiten der jeweiligen Kinderkliniken ermittelt. Eine Erinnerung zur Teilnahme wurde im Abstand von etwa 4 Wochen maximal 2-Mal zugeschickt, falls eine Abteilung noch nicht an der Umfrage teilgenommen hatte. Zur Erfassung der Teilnahme und zur Verhinderung von Mehrfachteilnahmen wurde jeder Intensivstation ein pseudonymisierter Schlüssel zugewiesen. Die Durchführung der Umfrage und das Speichern der Antworten erfolgte anonym, sodass Rückschlüsse auf die jeweiligen Intensivstationen im Nachhinein nicht möglich waren.

Die insgesamt 15 Umfrageelemente wurden von den Studienautoren erstellt und durch unabhängige Intensivmediziner hinsichtlich Verständlichkeit der Fragen, Eignung der Antworten und Einfachheit der Teilnahme überprüft. Der Fragebogen enthielt Nominal-, Kardinal- und offene Fragen. Die finale Version des Fragebogens ist als Anhang beigefügt.

Die statistische Auswertung erfolgte mit SigmaPlot (Version 12.5, Systat Software Inc., San Jose, CA, US). Nominaldaten werden als Zahlen und Prozent angegeben. Kardinaldaten wurden mittels t-Test verglichen und als Durchschnitt \pm Standardabweichung angegeben. In den Abbildungen werden Box-Whisker-Plots dargestellt. Die Box wird durch das obere und untere Quartil begrenzt, die Antennen bestimmen die 10. und 90. Perzentile, der Median ist als durchgehender Strich in der Box eingezeichnet. Eine Wahrscheinlichkeit mit p-Wert <0,05 wurde als statistisch signifikant definiert.

Das Studienprotokoll wurde von der lokalen Ethik-Kommission genehmigt (404/2017BO2).

\section{Ergebnisse}

Von den 583 kontaktierten Intensivstationen erhielten wir 283 Rückmeldungen (48,5\%). Davon entfielen 180 Rückmeldungen auf Deutschland (63,6\%, Rücklaufquote 58,3\%), 21 auf Österreich (7,4\%, Rücklaufquote 65,6\%), 16 auf die Schweiz (5,7\%, Rücklaufquote 69,6\%), 51 auf England (18\%, Rücklaufquote 30,4\%), 6 auf Frankreich $(2,1 \%$, Rücklaufquote $18,8 \%$ ) sowie 9 auf Norwegen $(3,2 \%$, 
Rücklaufquote 47,4\%). Die teilnehmenden Intensivstationen waren in Deutschland mehrheitlich gemischt neonatologisch-pädiatrisch ( $n=112,62,2 \%$ ), zu 33,3\% rein neonatologisch $(n=60)$ und zu $4,4 \%$ rein pädiatrisch $(n=8)$. Die Aufteilung der Intensivstationen in den anderen Ländern war wie folgt: 32,0\% gemischt neonatologisch-pädiatrisch $(n=33), 63,1 \%$ rein neonatologisch $(n=65)$ und $4,9 \%$ rein pädiatrisch $(n=5)$. Eine Intermediate Care Pflegestation (IMC) war in den teilnehmenden Kliniken aus Deutschland mehrheitlich nicht vorhanden ( $n=105,58,3 \%$ ). In 22 deutschen Kliniken (12,2\%) gab es eine gemischt neonatologisch-pädiatrische IMC, in 48 (26,7\%) eine rein neonatologische IMC und in $3(1,7 \%)$ eine rein pädiatrische IMC. Außerhalb Deutschlands gab es in 64 Kliniken eine IMC (62,1\%), 15 gemischt neonatologisch-pädiatrisch (14,6\%), 42 rein neonatologisch (40,7\%) sowie 7 rein pädiatrisch $(6,8 \%)$.

Die Anzahl an Intensivbehandlungsplätzen pro Station war in Deutschland im Vergleich zu den anderen teilnehmenden Ländern nicht unterschiedlich $(13,5 \pm 6,1$ gegenüber $15,1 \pm 11,3, p=0,12)$. Die besetzten Pflegestellen auf den Intensivstationen zeigten jedoch einen statistisch signifikanten Unterschied zwischen Deutschland und den anderen Ländern: die teilnehmenden deutschen Intensivstationen gaben an, dass 90,4\% der Stellen durchschnittlich be- setzt seien (SD 9,8\%). In den anderen Ländern waren zum Zeitpunkt der Umfrage 95,8\% (SD 7,2\%) der Pflegestellen besetzt ( $p<0,001$ ) ( $\vee$ Tab. 1, - Abb. 1). Ein anderes Bild zeigte sich hinsichtlich der besetzten Assistenzarzt- und Oberarztstellen: durchschnittlich waren 96,1\% (SD 9,4\%) der Assistenzarztstellen bzw. 95,7\% der Oberarztstellen (SD 12,1\%) in Deutschland besetzt gegenüber 91,9\% (SD 14,6\%) bzw. 90,9\% der Oberarztstellen (SD 14,6\%) in den anderen Ländern ( $p=0,03$ bzw. $p=0,04)$ ( $\triangleright$ Tab. 1).

Auf den pädiatrischen Intensivstationen von Zentren mit Kinderherzchirurgie zeigten sich ebenfalls signifikante Unterschiede: in Deutschland waren 87,7\% (SD 10,4\%) der Pflegestellen besetzt, in den anderen Ländern 95,7\% (SD 6,4\%, p =0,03). Ein ähnliches Bild zeigte der Blick auf Level-1-Perinatalzentren: hier waren in Deutschland 89,0\% (SD 10,3\%) der Pflegestellen besetzt, in den anderen Ländern 96,4\% (SD 6,8\%, p<0,001) ( $>$ Tab. 1).

Die Teilnehmer wurden gefragt, wie viele neonatologische bzw. pädiatrische Intensivpatienten in der Regel pro Pflegekraft versorgt werden, welches Verhältnis eigentlich vorgesehen ist und wie der Wunschversorgungsschlüssel der Abteilung wäre. Hier zeigten sich im neonatologischen Bereich signifikante Unterschiede ( $\triangleright$ Abb. 2). In Deutschland waren durchschnittlich 2,5 neonatologische $\mathrm{Pa}$ -

> Tab. 1 Unterschiede im Rücklauf der Fragebögen, der Intensivplätze und der Personalbesetzung zwischen Deutschland und anderen Ländern (Österreich, Schweiz, England, Frankreich, Norwegen).

\begin{tabular}{|c|c|c|c|}
\hline & & Deutschland & Andere \\
\hline \multicolumn{2}{|l|}{ Rücklauf } & $180 / 309(58,3 \%)$ & $103 / 274(37,6 \%)$ \\
\hline \multirow[t]{2}{*}{ Intensivplätze } & Durchschnitt \pm SD & $13,5 \pm 6,1$ & $15,1 \pm 11,3$ \\
\hline & Differenz (95\% KI), p-Wert & \multicolumn{2}{|c|}{$-1,6(-3,7,0,4), p=0,12$} \\
\hline \multirow[t]{2}{*}{ Pflegestellen } & Durchschnitt \pm SD & $90,4 \% \pm 9,8 \%$ & $95,8 \% \pm 7,2 \%$ \\
\hline & Differenz (95\% KI), p-Wert & \multicolumn{2}{|c|}{$-5,4 \%(-8,2 \%,-2,6 \%), p<0,001$} \\
\hline \multirow[t]{2}{*}{ AA-Stellen } & Durchschnitt \pm SD & $96,1 \% \pm 9,4 \%$ & $91,9 \% \pm 14,6 \%$ \\
\hline & Differenz (95\% KI), p-Wert & \multicolumn{2}{|c|}{$4,2 \%(0,3 \%, 8,2 \%), p=0,03$} \\
\hline \multirow[t]{2}{*}{ OA-Stellen } & Durchschnitt $\pm S D$ & $95,7 \% \pm 12,1 \%$ & $90,9 \% \pm 14,6 \%$ \\
\hline & Differenz (95\% KI), p-Wert & \multicolumn{2}{|c|}{$4,8 \%(0,3 \%, 9,4 \%), p=0,04$} \\
\hline \multirow[t]{2}{*}{ Pflegestellen Kardiochirurgie } & Durchschnitt $\pm S D$ & $87,7 \% \pm 10,4 \%$ & $95,7 \% \pm 6,4 \%$ \\
\hline & Differenz (95\% KI), p-Wert & \multicolumn{2}{|c|}{$-8,0 \%(-15,3 \%,-0,7 \%), p=0,03$} \\
\hline \multirow[t]{2}{*}{ Pflegestellen PNZ } & Durchschnitt \pm SD & $89,0 \% \pm 10,3 \%$ & $96,4 \% \pm 6,8 \%$ \\
\hline & Differenz (95\% KI), p-Wert & \multicolumn{2}{|c|}{$-7,5 \%(-11,4 \%,-3,5 \%), p<0,001$} \\
\hline
\end{tabular}
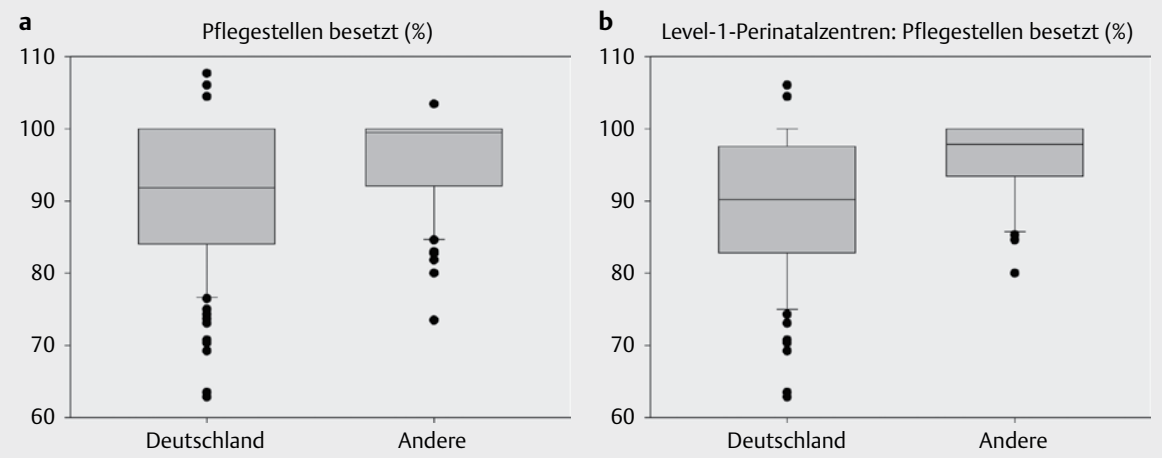

- Abb. 1 Besetzte Pflegestellen auf den neonatologischen und pädiatrischen Intensivstationen a sowie in Level-1-Perinatalzentren b im Vergleich Deutschland (DE) zu den anderen Ländern. 

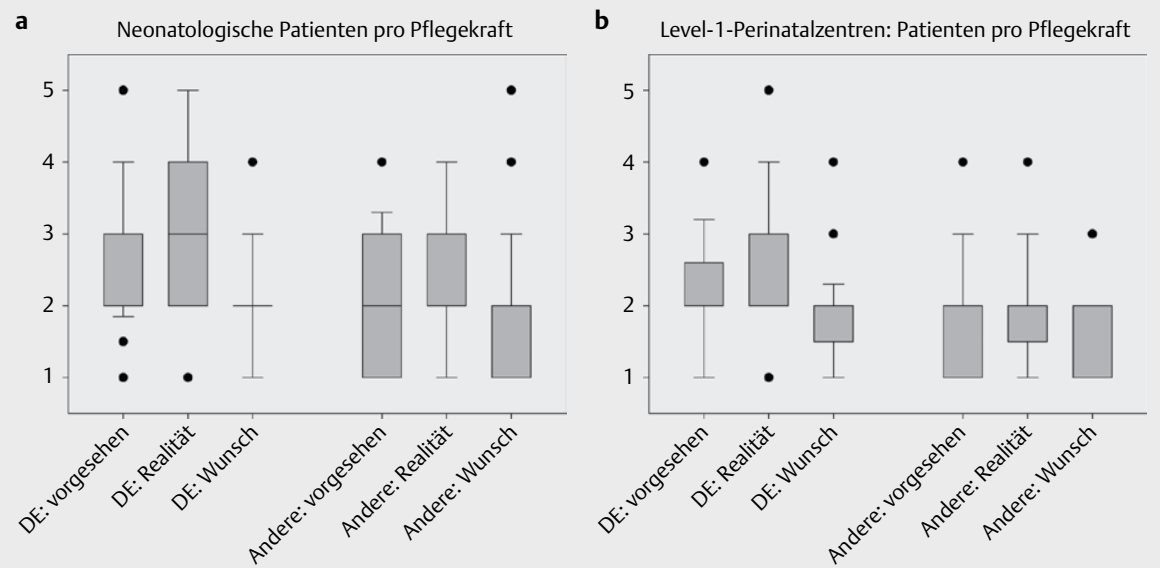

Abb. 2 Anzahl betreuter Patienten je Pflegekraft auf den neonatologischen Intensivstationen a sowie in Level-1-Perinatalzentren laut Stationsplanung, in der Realität und in der Wunschvorstellung im Vergleich Deutschland (DE) zu den anderen Ländern b.

a

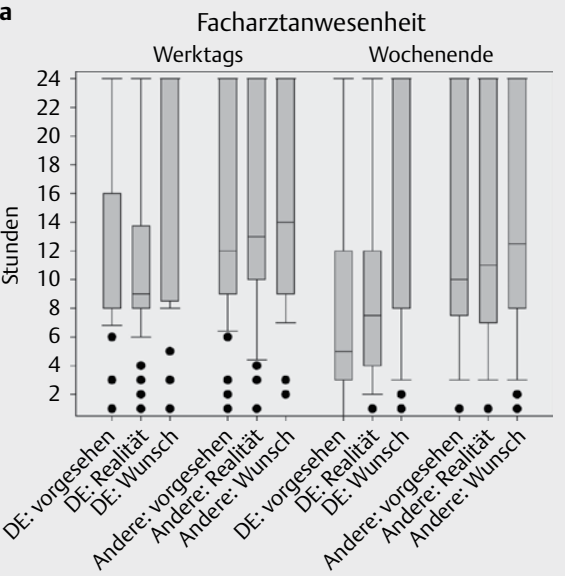

b

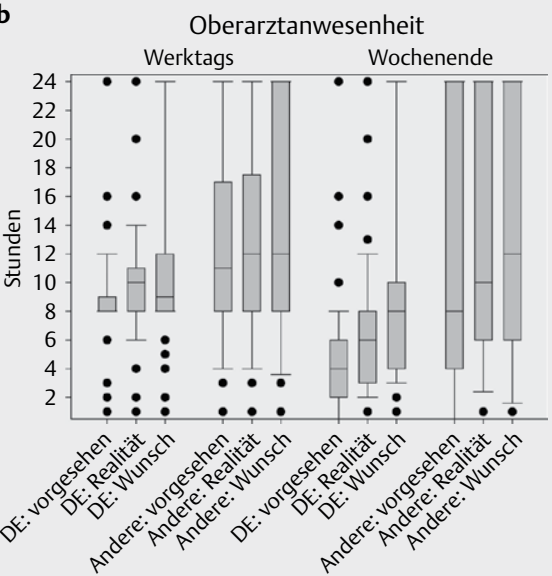

- Abb. 3 Anwesenheitsstunden pro Tag von Fachärzten a und Oberärzten b an Werktagen und am Wochenende laut Planung, in der Realität und in der Wunschvorstellung im Vergleich Deutschland (DE) zu den anderen Ländern.

tienten pro Pflegekraft vorgesehen (SD 1,1) gegenüber 2,1 (SD 0,9) in den anderen Ländern $(p=0,015)$. In der Realität wurden nach Angabe der Institutionen in Deutschland im Schnitt 3,0 neonatologische Patienten von einer Pflegekraft versorgt (SD 1,2) gegenüber 2,3 Patienten in den übrigen Ländern (SD 1,0, $p<0,001$ ). Die Wunschvorstellung ähnelt sich in Deutschland und den anderen Ländern (2,0 Patienten, SD 0,8 gegenüber 1,8 Patienten, SD 1,9, $\mathrm{p}=0,10)$. In der separaten Analyse von Level-1-Perinatalzentren ergaben sich ähnlich Unterschiede ( $\boldsymbol{A} \mathbf{A b b}$. 2): laut Planung sollten in Deutschland 2,3 Patienten (SD 0,99), in den anderen Ländern 1,8 (SD 0,78, $P=0,015$ ) von einer Pflegekraft versorgt werden. In der Realität waren es in Deutschland 2,7 (SD 1,0) gegenüber 2,0 Patienten im restlichen Kollektiv (SD 0,8, p<0,001). Die Wunschvorstellung lag in Deutschland bei 1,9 Patienten (SD 0,7 ) pro Pflegekraft und in den anderen Ländern bei 1,4 (SD 0,6, $p=0,003)$.

In der Versorgung auf pädiatrischen Intensivstationen waren in Deutschland ähnlich viele Patienten pro Pflegekraft vorgesehen wie in den anderen Ländern (2,5 Patienten, SD 1,4 gegenüber 2,5 Patienten, SD 2,1, p =0,99). In der Realität (2,9 Patienten, SD 1,7 gegenüber 2,3 Patienten, SD 1,7, $p=0,21$ ) sowie in der Wunschvorstellung (2,1 Patienten, SD 1,2 gegenüber 2,1 Patienten, SD 1,7, $\mathrm{p}=0,85$ ) ergaben sich hier keine signifikanten Unterschiede.

Auch die Analyse der ärztlichen Patientenversorgung erfolgte hinsichtlich der Planung, Realität und Wunschvorstellung. Hier zeigten sich jeweils keine statistisch signifikanten Unterschiede: Vorgesehen waren in Deutschland durchschnittlich 8,5 Intensivpatienten (SD 4,1) pro Arzt gegenüber 7,7 Patienten (SD 4,6) in den anderen Ländern ( $p=0,28)$, in der Realität fanden sich 9,0 Patienten (SD 4,2) gegenüber 7,8 Patienten (SD 5,2, $p=0,13$ ) und die Wunschvorstellung betrug 6,6 Patienten (SD 2,9) vs. 6,8 Patienten (SD 3,8, $p=0,78)$.

Zuletzt wurde noch die Facharzt- und Oberarztanwesenheit erfragt, wiederum laut Planung, in der Realität und nach Wunschvorstellung getrennt nach Werktagen und Wochenenden ( 
Im Facharztbereich zeigte sich, dass in Deutschland werktags die Anwesenheit eines Facharztes signifikant weniger geplant war als im Ausland (12,0 Stunden, SD 7,1 gegenüber 14,6 Stunden, SD 7,4, $p=0,04)$, wobei sich die Wunschvorstellung in beiden Erhebungsbereichen nicht signifikant unterschied (17,5 Anwesenheitsstunden, SD 7,7 gegenüber 16 Stunden, SD 8,0, p=0,31). In der Realität wurde die geplante Facharztanwesenheit bspw. durch ungeplante Überstunden nicht überschritten (Deutschland 11,3 Stunden, SD 6,2, $p=0,43$; andere Länder 14,8 Stunden, SD 7,2, p=0,86).

Die Situation am Wochenende stellte sich ähnlich dar: in Deutschland war durchschnittlich an 8,9 Stunden (SD 8,4) pro Tag die Anwesenheit eines Facharztes geplant, in den anderen Ländern an 13,2 Stunden (SD 8,4, p=0,003). Die reale Arbeitszeit verhielt sich hierzu ähnlich (Deutschland 8,9 Stunden, SD 7,1, p=0,97; andere Länder 13,5 Stunden, SD 8,4, p=0,88). Die Wunschvorstellung einer Facharztanwesenheit am Wochenende war wiederum länderübergreifend gleich: 15,9 Stunden (SD 8,8) gegenüber 15,1 Stunden (SD 8,4, $p=0,62$ ).

Im oberärztlichen Bereich zeichnete sich für die Werktage ein ähnliches Bild: in Deutschland war die Oberarztanwesenheit auf Intensivstationen signifikant weniger geplant als im Ausland (8,9 Stunden, SD 3,9 gegenüber 12,7 Stunden, SD 7,2, p<0,001). In der Realität fand sich kein signifikanter Unterschied zur Planung (Deutschland 9,7 Stunden, SD 4,2, p = 0,13; andere Länder 12,9 Stunden, SD 7,0, $p=0,87$ ). Die Wunschvorstellung für die Oberarztanwesenheit an den Werktagen war jedoch unterschiedlich: mit 11,0 Stunden (SD 5,7) in Deutschland gegenüber den anderen Ländern mit 14,1 Stunden (SD 8,0, p=0,007).

Am Wochenende zeigten sich wiederum signifikante Unterschiede für die geplante Oberarztanwesenheit pro Tag (Deutschland 5,2 Stunden, SD 5,1 gegenüber 11,3 Stunden, SD 8,7, $\mathrm{p}<0,001)$. Die geplante Anwesenheitszeit wurde in Deutschland nicht eingehalten (6,6 Stunden, SD 5, $1, p=0,049$; andere Länder $12,3$ Stunden, SD 8,3, $p=0,56)$. Die Wunschvorstellung für die Oberarztanwesenheit am Wochenende war wiederum unterschiedlich: Deutschland 8,6 Stunden (SD 6,5) gegenüber 13,2 Stunden (SD 8,5, p<0,001).

Die Facharztanwesenheit auf Intensivstationen von Zentren mit kinderherzchirurgischer Abteilung lag in Deutschland werktags laut Planung bei 16,3 Stunden (SD 7,7), am Wochenende bei 15,6 Stunden (SD 9,1). Dies entsprach auch der Realität mit werktags 15,2 Stunden (SD 7,2) und am Wochenende 14,0 Stunden (SD 8,5). In Level-1-Perinatalzentren sind werktags durchschnittlich 13,4 Stunden (SD 6,7) mit Facharztanwesenheit geplant, am Wochenende 10,6 Stunden (SD 8,7). In der Realität wurden diese geplanten Zahlen eingehalten: werktags 12,5 Stunden (SD 5,6), am Wochenende 10,7 Stunden (SD 6,8).

\section{Diskussion}

Unsere Umfrage zeigt auf neonatologischen und pädiatrischen Intensivstationen in Deutschland im Vergleich zu anderen Ländern (Österreich, Schweiz, England, Frankreich und Norwegen) personelle Unterschiede sowohl im pflegerischen wie auch im ärztlichen Bereich auf. Unsere Daten unterstreichen die Notwendigkeit der aktuell geführten Diskussionen über Wege aus dem Pflegenotstand in Deutschland. Wir fanden einen signifikanten Nachteil hinsicht- lich der besetzten Pflegestellen im Vergleich zu den anderen Ländern, die auch in den Spezialpflegebereichen der Kinderkardiochirurgie und Level-1-Perinatalmedizin bestehen. Dies macht sich bei den teilnehmenden Kliniken in der Anzahl an Patienten bemerkbar, die von einer Pflegekraft versorgt werden müssen. In der Neonatologie (sowohl in der Gesamtheit wie auch in den Level-1Perinatalzentren) werden laut Stationsplanung und noch mehr in der Realität signifikant mehr Patienten von einer Pflegekraft versorgt als in den anderen befragten Ländern. Der Unterschied zwischen Planung und Realität wird bspw. durch Krankheit, Fehlzeiten und Ausfall bei Überbelastung verursacht, wodurch die personelle Belastungssituation aggraviert wird und dieser Umstand mutmaßlich weitere Ausfälle zur Folge hat. Unsere Ergebnisse liegen ebenfalls über den Empfehlungen der Deutschen Interdisziplinären Vereinigung für Intensiv- und Notfallmedizin (DIVI), die zum Schluss kommt, dass maximal 2 Intensivpatienten von einer Pflegekraft versorgt werden sollen [15]. Auch wenn diese Untersuchung nicht explizit die Einhaltung der Pflegevorgaben hinsichtlich des Pflegeschlüssels des G-BA für den Bereich Neonatologie erfragt hat, erscheint es, dass die Umsetzung der Vorgabe Schwierigkeiten bereiten dürfte, dass eine Pflegekraft in Level-1-Perinatalzentren maximal 2 intensivüberwachungspflichtige Frühgeborene mit Geburtsgewicht von weniger als $1500 \mathrm{~g}$ betreuen darf [9].

Unabhängig von den Richtlinien mehren sich Hinweise, dass eine Unterbesetzung im Pflegebereich, egal ob subjektiv von den Mitarbeitern empfunden oder objektivierbar, zu einer niedrigeren $\mathrm{Ar}$ beitszufriedenheit und erhöhtem negativem Stressempfinden führt $[13,25]$.

Darüber hinaus kommt es aufgrund von Pflegemangel zwangsläufig zu Sperrung von Intensivbetten, die eine Beeinträchtigung der Notfallversorgung und Einschränkung der Operationskapazitäten nach sich zieht. In der Arbeit von Karagiannidis et al. wurde aufgezeigt, dass in 76\% der befragten Intensivstationen in Deutschland in unterschiedlichem Maße Intensivbetten meist aufgrund von Pflegemangel gesperrt wurden [17]. Als Ursachen für den Pflegemangel wurden unter anderem eine inadäquate Vergütung für Fachpflegekräfte für Intensivmedizin, fehlende Aufstiegsmöglichkeiten sowie eine hohe psychische und physische Belastung im Beruf aufgeführt. Ein ähnliches Bild zeigte eine aktuelle Umfrage der DIVI unter deutschen Kinderintensivstationen: im Mittel waren rund $20 \%$ der möglichen Intensivbetten wegen fehlender Pflegekräfte gesperrt, sodass regelmäßig Kinder abgelehnt werden mussten [6].

Für die Patienten bedeutet eine Unterbesetzung in der Pflege einen potenziellen Qualitätsverlust in der Versorgung. Eine (subjektive) hohe Arbeitsbelastung kann sich ähnlich wie eine Verlängerung der Arbeitszeit negativ auf die Patientenpflege und das Erkennen bedrohlicher Situationen auswirken [4, 5, 26]. Studien aus der Erwachsenenmedizin und der Pädiatrie zeigten eine gesteigerte Prävalenz von Hygienemängeln, beatmungsassoziierten Pneumonien, eine höhere Rate von fehlgeschlagenen Extubationsversuchen sowie eine gesteigerte Morbidität und Mortalität [2, 3, 12, $14,18,20,23,29]$.

Zusätzlich zu dem Mangel im pflegerischen Bereich lag die Facharzt- und Oberarztanwesenheit sowohl werktags als auch am Wochenende in Deutschland deutlich niedriger als in den anderen befragten europäischen Ländern. Es gibt Hinweise, dass die Betreu- 
ung durch Fach- und Oberärzte mit dem Behandlungserfolg korreliert $[11,16]$. Durch die im Vergleich niedrigere Anwesenheit von Intensivmedizinern und damit fehlender Expertise wird der durch den Pflegemangel einhergehende mögliche Qualitätsverlust in der Patientenversorgung zusätzlich verschärft. Dies betrifft auch den hochspezialisierten Bereich der kinderkardiochirurgischen Intensivstationen.

Um Auswege aus dem Personalmangel insbesondere im pflegerischen Bereich zu finden, müssen unserer Meinung nach die Arbeitsbedingungen seitens der Gesundheitspolitik und Klinikleitungen dringend optimiert werden. Höhere Vergütung, flexiblere Arbeitszeitmodelle, Aufstiegsmöglichkeiten, Zusatzqualifikationen und Übernahme von definierten Verantwortungsbereichen könnten hierzu beitragen [17]. Zur Rekrutierung und Bindung qualifizierter Ärzte erscheint die Auflockerung festgefahrener hierarchischer Strukturen, Förderung von Teamarbeit, Abschaffung von Bürokratie zugunsten der Patientenversorgung, Bekämpfung ausufernder sowie unflexibler Arbeitszeiten, Möglichkeiten zur Kinderbetreuung und damit besserer Vereinbarkeit von Familie und Beruf essenziell. Die Erhaltung der psychischen und physischen Gesundheit der Mitarbeiter muss im Fokus stehen, um die zunehmenden Belastungs- und Stresssituationen bewältigen zu können [22]. Einen Beitrag hierzu könnten bauliche Maßnahmen zur Verbesserung des Stationsdesigns sowie zur Schaffung einer effizienten und ressourcenschonenden Arbeitsatmosphäre leisten. Eine Stressoren- und Lärmreduktion scheint nicht nur für die Genesung der pädiatrischen Patienten von Vorteil zu sein, sondern auch das Stressempfinden der Mitarbeiter zu reduzieren [19].

\section{Limitationen der Studie}

Als Limitation dieser Untersuchung muss der Rücklauf von nur knapp der Hälfte der befragten Abteilungen genannt werden, wobei die Antwortrate in Deutschland mit fast zwei Dritteln deutlich darüber liegt. Warum insbesondere der Rücklauf von den französischen und englischen Kliniken relativ gering war, bleibt Spekulation. Eventuell ist dort der Fachkräftemangel noch nicht so ausgeprägt oder nicht Gegenstand aktueller Diskussionen, weshalb das Interesse an einer Teilnahme an dieser Befragung weniger ausgeprägt war. Prinzipiell war es auch schwieriger an persönliche Kontaktdaten englischer Abteilungsleiter zu kommen, da diese nur selten öffentlich zugänglich waren. Somit fand der Kontakt über allgemeine Klinik-E-Mailadressen statt, was möglicherweise ebenfalls zum schlechten Rücklauf aus England beigetragen.

Bereits während der Durchführung der Umfrage wurde insbesondere von neonatologischen Zentren bemängelt, dass die Angabe, wie viele Patienten von einer Pflegekraft versorgt werden, nicht in Einklang mit der aktuellen GB-A Vorgabe sind, in der Unterschiede zwischen invasiv-beatmeten, nicht-invasiv-beatmeten und anderen Kindern gemacht werden. Wir hatten uns explizit gegen eine derartige Unterteilung entschieden, da die Erstellung eines Dienstplanes in der Regel Wochen bis Monate im Voraus erfolgt und Fluktuationen der Patientenanzahl und der Schweregrade der Erkrankungen auf einer neonatologischen Intensivstation nur bedingt abdecken kann. Die nächtliche Geburt eines schwerstkranken asphyktischen Kindes kann meist nicht sofort durch Entlassung/ Verlegung anderer Patienten oder sofortiger Hinzunahme weiterer Pflegekräfte kompensiert werden. Um den Fragebogen so einfach und kurz wie möglich zu halten, sollten die teilnehmenden Abtei- lungen im Fragebogen einen Wert eintragen, der diesen Gegebenheiten am nächsten kommt.

\section{Schlussfolgerung}

Im Vergleich mit verschiedenen europäischen Ländern zeigt sich auf neonatologischen und pädiatrischen Intensivstationen ein Fachkräftemangel im pflegerischen Bereich. Die Entscheidungsträger in Politik und den Einrichtungen sind gefragt, Strategien zur vermehrten Ausbildung, Einstellung und Bindung von Pflegekräften zu entwickeln.

\section{Stellungsnahme zur Autorenschaft}

Contribution to study concept and design: J. Michel, M. Hofbeck, F. Neunhoeffer acquisition of data: J. Michel, L. Gerster analysis and interpretation of data: J. Michel, L. Gerster, F. Neunhoeffer drafting the manuscript: J. Michel, L. Gerster, F. Neunhoeffer revising the manuscript: J. Michel, M. Hofbeck, L. Gerster, F. Neunhoeffer

\section{Interessenkonflikt}

Die Autoren geben an, dass kein Interessenkonflikt besteht.

Literatur

[1] Aiken LH, Sloane DM, Bruyneel L et al. Nurse staffing and education and hospital mortality in nine European countries: a retrospective observational study. Lancet (London, England) 2014; 383: 1824-1830

[2] Beltempo M, Lacroix G, Cabot M et al. Association of nursing overtime, nurse staffing and unit occupancy with medical incidents and outcomes of very preterm infants. J Perinatol 2018; 38: 175-180

[3] Bischoff P, Geffers C, Gastmeier P. Hygiene measures in the intensive care station. Medizinische Klinik, Intensivmedizin und Notfallmedizin 2014; 109: 627-639

[4] Cooke CR, Watkins TR, Kahn JM et al. The effect of an intensive care unit staffing model on tidal volume in patients with acute lung injury. Critical care (London, England) 2008; 12: R134

[5] Dall'Ora C, Griffiths P, Redfern O et al. Nurses' 12-hour shifts and missed or delayed vital signs observations on hospital wards: retrospective observational study. BMJ open 2019; 9: e024778

[6] DIVI. DIVI kritisiert Personalmangel auf Kinderintensivstationen in Deutschland. In: 20.11.2018

[7] Driscoll A, Grant M], Carroll D et al. The effect of nurse-to-patient ratios on nurse-sensitive patient outcomes in acute specialist units: a systematic review and meta-analysis. European journal of cardiovascular nursing: journal of the Working Group on Cardiovascular Nursing of the European Society of Cardiology 2018; 17: 6-22

[8] GBA. Gemeinsamer Bundesausschuss. Richtlinie über Maßnahmen zur Qualitätssicherung der herzchirurgischen Versorgung bei Kindern und Jugendlichen gemäß § 136 Absatz 1 Nummer 2 SGB V. In: 05.12.2018

[9] GBA. Gemeinsamer Bundesausschuss. Richtlinie über Maßnahmen zur Qualitätssicherung der Versorgung von Früh- und Reifgeborenen. In: 17.05.2018

[10] Griffiths P, Ball J, Bloor K et al. Health Services and Delivery Research. In: Nurse staffing levels, missed vital signs and mortality in hospitals: retrospective longitudinal observational study. Southampton (UK): NIHR Journals Library 2018 
[11] Gupta P, Rettiganti M, Rice TB et al. Impact of 24/7 In-Hospital Intensivist Coverage on Outcomes in Pediatric Intensive Care. A Multicenter Study. American journal of respiratory and critical care medicine 2016; 194: 1506-1513

[12] Isfort M. Influence of personnel staffing on patient care and nursing in German intensive care units. Descriptive study on aspects of patient safety and stress indicators of nursing. Medizinische Klinik, Intensivmedizin und Notfallmedizin 2013; 108: 71-77

[13] Isfort M. Evaluation der Pflegebedingungen auf Intensivstationen. ProCare 2018; 23: 20-25

[14] Jansson MM, Syrjala HP, Ala-Kokko TI. Association of nurse staffing and nursing workload with ventilator-associated pneumonia and mortality: a prospective, single-center cohort study. The Journal of hospital infection 2019; 101: 257-263

[15] Jorch G, Kluge S, König F et al. Empfehlungen zur Struktur und Ausstattung von Intensivstationen. DIVI 2011; 2: 1-40

[16] Kahn JM, Brake H, Steinberg KP. Intensivist physician staffing and the process of care in academic medical centres. Quality \& safety in health care 2007; 16: 329-333

[17] Karagiannidis C, Kluge S, Riessen R et al. Auswirkungen des Pflegepersonalmangels auf die intensivmedizinische Versorgungskapazität in Deutschland 2018, doi:10.1007/s00063-018-0457-3

[18] Kochanek M, Boll B, Shimabukuro-Vornhagen A et al. Staffing needs of an intensive care unit in consideration of applicable hygiene guidelines - an exploratory analysis. Deutsche medizinische Wochenschrift 1946; 2015: e136-e141

[19] Kudchadkar SR, Beers MC, Ascenzi JA et al. Nurses' Perceptions of Pediatric Intensive Care Unit Environment and Work Experience After Transition to Single-Patient Rooms. American journal of critical care: an official publication, American Association of Critical-Care Nurses 2016; 25: e98-e107

[20] Nogueira TDA, Menegueti MG, Perdona G et al. Effect of nursing care hours on the outcomes of Intensive Care assistance. PloS one 2017; 12: e0188241
[21] PEDIATRICS AAO. Joint policy statement - guidelines for care of children in the emergency department. Journal of emergency nursing: JEN: official publication of the Emergency Department Nurses Association 2013; 39: 116-131

[22] Rodriguez-Rey R, Palacios A, Alonso-Tapia J et al. Are Pediatric Critical Personnel Satisfied With Their Lives? Prediction of Satisfaction With Life From Burnout, Posttraumatic Stress, and Posttraumatic Growth, and Comparison With Noncritical Pediatric Staff. Pediatric critical care medicine: a journal of the Society of Critical Care Medicine and the World Federation of Pediatric Intensive and Critical Care Societies 2019; 20: e160-e169

[23] Rooney SR, Donohue JE, Bush LB et al. Extubation Failure Rates After Pediatric Cardiac Surgery Vary Across Hospitals. Pediatric critical care medicine: a journal of the Society of Critical Care Medicine and the World Federation of Pediatric Intensive and Critical Care Societies 2019, doi:10.1097/pcc.0000000000001877

[24] Rosenberg DI, Moss MM. Guidelines and levels of care for pediatric intensive care units. Critical care medicine 2004; 32: 2117-2127

[25] Torre M, Santos Popper MC, Bergesio A. Burnout prevalence in intensive care nurses in Argentina. Enfermeria intensiva 2018, doi:10.1016/j. enfi.2018.04.005

[26] Tubbs-Cooley HL, Mara CA, Carle AC et al. Association of Nurse Workload With Missed Nursing Care in the Neonatal Intensive Care Unit. JAMA pediatrics 2019; 173: 44-51

[27] Valentin A, Ferdinande P. Recommendations on basic requirements for intensive care units: structural and organizational aspects. Intensive care medicine 2011; 37: 1575-1587

[28] Warncke G, Hoffmann F, Sasse M et al. A multinational survey on the infrastructural quality of paediatric intensive care units 2018; 8: 105

[29] West E, Barron DN, Harrison D et al. Nurse staffing, medical staffing and mortality in Intensive Care: An observational study. International journal of nursing studies 2014; 51: 781-794 